HU-EP-04/18

hep-th/0403220

\title{
On supersymmetric solutions of type IIB supergravity with general fluxes
}

\author{
Gianguido Dall'Agata \\ Humboldt Universität zu Berlin, Institut für Physik, \\ Newtonstrasse 15, D-12489 Berlin, Germany \\ E-mail: dallagat@physik.hu-berlin.de
}

\begin{abstract}
We propose a general spinor Ansatz to find supersymmetric configurations preserving 4-dimensional Poincaré invariance in the context of type IIB supergravity in the presence of general fluxes. We show how this removes the imaginary-selfduality (ISD) constraint on the 3 -form flux and present a simple example with nonvanishing $(0,3)$ flux. To characterize the geometrical properties of such configurations we will use the tool of $\mathrm{SU}(2)$ structures on the internal space, which are naturally linked to the form of the Ansatz we propose.
\end{abstract}




\section{Introduction}

The analysis of supersymmetric backgrounds in the presence of fluxes has a prominent role in addressing various longstanding problems of string theory like moduli stabilization. Vacua preserving 4-dimensional Poincaré invariance are especially interesting because they can be used either in string compactifications or in the context of the gauge/gravity correspondence.

In this paper we will focus on $\mathcal{N}=1$ solutions of type IIB supergravity and give a geometrical characterization of these type of backgrounds for generic configurations of fluxes. In order to do so we are going to use the tool of group structures on the internal manifold. This powerful method gives a systematic way to translate supersymmetry conditions in terms of differential constraints on some structures on the internal manifold, which then define the metric and flux. This type of analysis, first introduced in [1, has been very fruitful in constructing and classifying new solutions in the context of string theory [2] 13 (For reviews on string theory solutions with fluxes see also [14, 15]). It has been especially emphasized in [7] that the choice of an appropriate spinor Ansatz is strictly related to the possible group structure existing on a manifold and may affect the form of the resulting solutions.

Looking for $\mathcal{N}=1$ solutions of type IIB theory, three different types of supersymmetry parameters were used. All these assume that there is one globally defined spinor on the internal manifold $\eta_{-}$(with its complex conjugate $\eta_{+}$) so that the 10-dimensional supersymmetry parameter $\epsilon$ reads

$$
\begin{array}{ll}
\text { Type A: } & \epsilon=a \varepsilon \otimes \eta_{-}+a^{*} \varepsilon^{*} \otimes \eta_{+}, \\
\text {Type B: } & \epsilon=a \varepsilon \otimes \eta_{-}, \\
\text {Type C: } & \epsilon=a \varepsilon \otimes \eta_{-}+b \varepsilon^{*} \otimes \eta_{+},
\end{array}
$$

with $a$ and $b$ complex functions, $\varepsilon$ is the 4-dimensional supersymmetry parameter and $\eta_{ \pm}$ are normalized to 1. Type A Ansatz was first introduced by Strominger in the context of the heterotic theory [16], applied to type IIB in [17] and recently also considered in [18, 3, 5, 19, 20]. Type B, named after [21], was utilized in the type IIB context in [22][26]. Finally, a spinor of the form (1.3) was proposed in [27] as a way to construct solutions interpolating between $\mathrm{A}$ and $\mathrm{B}$ types. We name it type $\mathrm{C}$, being the third different type of Ansatz used so far, though this should not be confused with the S-dual of type A which is named in the same way in [27].

Based on the existence of a single globally defined spinor, (1.1)-(1.3) imply the existence of an $S U(3)$ structure on the internal manifold. This structure is characterized by an almost complex structure $J$ and a 3 -form $\Omega$ which are naturally given in terms of $\eta_{ \pm}$:

$$
J_{m n}=-i \eta_{-}^{T} \gamma_{m n} \eta_{+}, \quad \Omega_{m n p}=-\eta_{+}^{T} \gamma_{m n p} \eta_{+} .
$$


The supersymmetry conditions following from (1.1)-(1.3) using a metric and a 5-form preserving Poincaré symmetry, impose that the allowed supersymmetric 3-form fluxes have to be only of type $(2,1)$ or $(1,2)$ with respect to the natural complex structure $J$. For type B the requirement is even stronger, because only fluxes which are of type $(2,1)$ and primitive with respect to $J$ are allowed [25, 26].

There are however solutions in the literature which hint to the possibility of supersymmetric $\mathcal{N}=1$ solutions of type IIB string theory in which the flux may contain also $(3,0)$ and $(0,3)$ contributions 24, 28]. For this reason one should consider a more general Ansatz for the form of the supersymmetry parameter which could provide these solutions. A general Ansatz which gives the desired result is

$$
\text { Type D: } \quad \epsilon=a \varepsilon \otimes \eta_{-}+\varepsilon^{*} \otimes\left(b \eta_{+}+c \chi_{+}\right),
$$

where a new spinor $\chi$, orthogonal to $\eta$, is introduced. This imposes some strong restriction on the possible choice of internal manifold. One is indeed allowed to employ such an Ansatz only if the internal manifold has an $S U(2)$ structure and therefore admits two globally defined spinors $^{1}$. Of course, since an $S U(2)$ structure can be embedded in different ways inside an $S U(3)$ structure there is no more the notion of a "natural" complex structure with respect to which one defines the Hodge decomposition of forms. There is actually a $U(1)$-worth of different almost complex structures, which therefore can be parameterized by a phase. Anyway, once a choice is made, one can see that it is possible to combine $(3,0)$ and/or $(0,3)$ fluxes with supersymmetry. Also the statements about the integrability of such a structure will depend on the phase choice.

Let us stress here that although we look for $\mathcal{N}=1$ solutions, the configurations we obtain may preserve more supersymmetry. Already in the case of a strict $S U(3)$ structure, namely an internal manifold allowing for just one globally defined spinor, supersymmetric configurations may preserve $\mathcal{N}=2$. This happens for instance in the degenerate case of zero fluxes, where the group structure is identified with the holonomy of the internal manifold, which is then Calabi-Yau. This, of course, is even more true in the case of $S U(2)$ structures.

It is interesting to notice that, due to the $S U(2)$ structure, the internal manifold will always admit an almost product structure which allows us to discuss them as fibrations of 2-manifolds on 4-manifolds. In simple cases this structure is even integrable, therefore simplifying the analysis and allowing to exhibit solutions in closed form. In this paper we will discuss the form of the $\mathrm{SU}(2)$ structures allowed by supersymmetry when the type D Ansatz (1.5) is used for the spinor parameter. We will show that the generic internal manifold

\footnotetext{
${ }^{1}$ It should be remembered that although the two spinors are orthogonal they are not independent, but, as we will show later, they are related through the $S U(2)$ structure tensors. This also explains why seemingly different Ansätze like in [28] fall instead in our classification.
} 
may be non-complex and indeed allows for $(3,0)$ or $(0,3)$ fluxes. As an application we will provide a simple solution with $(0,3)$ flux and holomorphic dilaton. This is a warped product of Minkowski space-time with an internal manifold which is an $\mathbb{R}^{2}$ fibration over a $K 3$.

After this introduction, in section 2 we will discuss in detail the spinor Ansatz and its relation to both $S U(3)$ and $S U(2)$ structures. Then, in section 3, we will perform a detailed analysis of the supersymmetry conditions reinterpreting them in terms of the $S U(2)$ structures. Finally, we will show how to produce simple solutions with $(3,0)$ and $(0,3)$ fluxes in section 4 and conclude with some comments in section 5 . We also give an appendix with more details on conventions, notations and $S U(2)$ structures in 6 dimensions.

\section{Supersymmetry Ansatz and group structures}

\subsection{Preliminaries}

Type IIB supersymmetry transformations [29, 30] read

$$
\begin{aligned}
\delta \psi_{M} & =\frac{1}{\kappa}\left(D_{M}-\frac{i}{2} Q_{M}\right) \epsilon+\frac{i}{480} \Gamma^{M_{1} \ldots M_{5}} F_{M_{1} \ldots M_{5}} \gamma_{M} \epsilon-\frac{1}{16} \Gamma_{M} G \epsilon^{*}-\frac{1}{8} G \Gamma_{M} \epsilon^{*}, \\
\delta \lambda & =\frac{i}{\kappa} \Gamma^{M} P_{M} \epsilon^{*}-\frac{i}{4} G \epsilon
\end{aligned}
$$

where we used the contraction $G \equiv 1 / 6 G_{M N P} \Gamma^{M N P}$. Here and in the following we will use the conventions of [30, where the supersymmetry conditions and covariant equations of motion for type IIB supergravity were first derived ${ }^{2}$. The definition of the dilaton-axion curvature $P_{M}=f^{2} \partial_{M} B$ and $U(1)$ connection $Q_{M}=f^{2} \operatorname{Im}\left(B \partial_{M} B^{*}\right)$ can be related to the standard stringy quantities $\tau=C+i e^{-\phi}$ by using $B=\frac{1+i \tau}{1-i \tau}$ and $f^{2}=1 /\left(1-|B|^{2}\right)$. The 3-form field strength $G=f\left(F_{3}-B F_{3}^{*}\right)$, where $F_{3}=d A_{2}$, follows from a complex 2-form $A_{2}$ which is related to the usual string NS and RR forms as $\kappa A_{2}=g\left(B_{N S}+i B_{R R}\right)$. The Bianchi identities for these forms are

$$
\begin{aligned}
d P & =2 i Q \wedge P, \\
d G & =i Q \wedge G+\bar{G} \wedge P, \\
d F_{5} & =i \frac{\kappa}{8} G \wedge \bar{G},
\end{aligned}
$$

together with the connection which satisfies $d Q=i \bar{P} \wedge P$. All the spinors are complex Weyl spinors satisfying $\Gamma^{11} \psi_{M}=-\psi_{M}, \Gamma^{11} \lambda=\lambda$ and $\Gamma^{11} \epsilon=-\epsilon$.

As said in the introduction, we are looking for solutions which preserve 4-dimensional Poincaré invariance. This type of solutions can be used for compactifications of type IIB

\footnotetext{
${ }^{2}$ For a discussion and derivation of a covariant action see [31, 32].
} 
supergravity as well as for the gauge/gravity correspondence. It should indeed be noticed that $A d S_{5}$ can be written as an $\mathbb{R}$ foliation of 4-dimensional Minkowski spacetime and that the domain-wall solutions describing holographic renormalization group flows are also warped products of 4-dimensional Minkowski space with $\mathbb{R}$ and some 5-dimensional compact space which, together, define a non compact 6-dimensional internal manifold. For the 10dimensional metric we will then take a warped product of 4-dimensional Minkowski space and some internal 6-dimensional euclidean manifold [26]

$$
d s^{2}=\frac{1}{\sqrt{Z(y)}} \eta_{\mu \nu} d x^{\mu} \otimes d x^{\nu}+\sqrt{Z(y)} g_{m n}(y) d y^{m} \otimes d y^{n}
$$

In order to preserve 4-dimensional Poincaré invariance on the full solution we also ask that the RR 5-form satisfies

$$
F_{0123 m}=\partial_{m} h(y)
$$

as well as the self-duality property $* F_{5}=F_{5}$. We do not impose any restriction on the dilaton or on the 3-form flux.

Before solving the supersymmetry conditions one needs to further specify an Ansatz for the spinor parameter and therefore we will now discuss more in detail how we arrived to the (1.5) Ansatz.

\subsection{Spinor Ansatz}

Since we are looking for solutions preserving 4-dimensional Poincaré invariance, we will use this property to perform a " $4+6$ " splitting of the supersymmetry spinor as well as of the 10-dimensional $\Gamma$ matrices. For generic $\mathcal{N}=1$ solutions this implies that the 10 -dimensional supersymmetry parameter gets factorized

$$
\epsilon(x, y)=\varepsilon(x) \otimes \zeta^{1}(y)+\varepsilon^{*}(x) \otimes\left(\zeta^{2}(y)\right)^{*},
$$

where $\varepsilon$ is a Weyl spinor on Minkowski space-time and $\zeta^{1}, \zeta^{2}$ are generic 6 -dimensional chiral spinors on the internal manifold.

In order to explicitly solve the supersymmetry equations it is important to establish the properties and relations between these spinors. First one should note that due to the chirality properties of the 10- and 4-dimensional spinors $\Gamma^{11} \epsilon(x, y)=-\epsilon(x, y), \gamma^{5} \varepsilon(x)=\varepsilon(x)$ and the definition of the 10-dimensional $\Gamma$ matrices in terms of the lower dimensional ones (see the appendix for more details), $\zeta^{i}$ should have a definite chirality $\gamma^{7} \zeta^{i}=-\zeta^{i}$. More restrictions can then follow by assuming that these 2 spinors are not unrelated, as in [17, 26, 27]. Generically, a globally well defined supersymmetric solution implies that the aforementioned

spinors are also globally defined and, accordingly, that the structure group of the tangent 
bundle over the internal manifold is reduced. The group of transformations $G$ required to patch the tangent bundle over the manifold is not the generic $S O(6)$ group of a Riemannian six-manifold, but a subgroup of it, $G \subset S O(6)$. The spinors which are globally defined, those which define our supersymmetry parameters, must not transform under $G$ and therefore are singlets under the $S O(6) \rightarrow G$ decomposition. When $\zeta^{1}$ and $\zeta^{2}$ are chosen parallel, or one of the two is vanishing, then the internal manifold displays an $S U(3)$ structure. Decomposing $S O(6) \simeq S U(4) \rightarrow S U(3)$, we see that the spinorial representation admits one singlet $4 \rightarrow 3+1$. This implies that an $S U(3)$ structure admits just one globally defined complex chiral spinor which is to be identified with the surviving supersymmetry parameter $\zeta$. Since this latter is globally defined, we can always normalize it to 1 , or extract from it the normalized spinor, say $\eta_{-}$. The subscript refers to the 6 -dimensional chirality $\gamma^{7} \eta_{ \pm}= \pm \eta_{ \pm}$. The general form of the supersymmetry parameter for solutions with an internal manifold preserving an $S U(3)$ structure is then

$$
\epsilon(x, y)=\varepsilon(x) \otimes a(y) \eta_{-}(y)+\varepsilon^{*}(x) \otimes b(y) \eta_{+}(y),
$$

where $a, b$ are complex functions and $\eta_{+}=\eta_{-}^{*}$. This includes all the solutions presented in the paper [27] and more because we allow for the norms of $a$ and $b$ to be unrelated. For $a=b^{*}$ we recover the type A Ansatz (1.1) and for $b=0$ we recover the B Ansatz (1.2). The functions $a$ and $b$ need not be related, though. The 10-dimensional supersymmetry parameter is a Weyl spinor, which can be written as the sum of 2 real Majorana-Weyl spinors: $\epsilon=\epsilon_{1}+i \epsilon_{2}$. Having an $S U(3)$ structure means that we can write them as

$$
\epsilon_{i}=\varepsilon \otimes f_{i} \eta_{-}+\varepsilon^{*} \otimes f_{i}^{*} \eta_{+}
$$

where $f_{i}(y)$ are arbitrary complex functions. This finally implies that $a=f_{1}+i f_{2}$ and $b=f_{1}^{*}+i f_{2}^{*}$ which therefore are generically unrelated complex functions too.

The $S U(3)$ structure can also be described [33] by an almost complex structure $J$ and a globally defined 3 -form $\Omega$. These are also singlets under the $S O(6) \rightarrow S U(3)$ decomposition and arise in the product of the fundamental invariant spinors:

$$
\begin{aligned}
J_{m n} & =-i \eta_{-}^{T} \gamma_{m n} \eta_{+}, \\
\Omega_{m n p} & =-\eta_{+}^{T} \gamma_{m n p} \eta_{+} .
\end{aligned}
$$

By using these tensors in the analysis of the supersymmetry conditions, once contracted with $\eta_{ \pm}$, one can extract quite easily the conditions

$$
\Omega \wedge G=0=\bar{\Omega} \wedge G .
$$

This means that having an $S U(3)$ structure on the internal space, which forces the (2.9) spinor Ansatz, one can only use 3 -form fluxes which are of $(2,1)+(1,2)$ type with respect 
to the natural complex structure defined by the covariantly constant spinor $\eta_{ \pm}$. Any $(3,0)$ or $(0,3)$ flux with respect to $J$ defined by (2.11) breaks supersymmetry.

A possible wayout is to make a stronger requirement on the structure of the internal manifold and ask for an $S U(2)$ structure. The existence of an $S U(2)$ structure on the internal manifold implies the possibility of having an additional globally defined spinor $\chi$, which may be used to modify (2.9) . There are indeed 2 singlets in the decomposition of the fundamental representation of $S U(4)$ under $S U(2)$. Taking again $\chi$ to be canonically normalized and using the same conventions as for $\eta$, we can generically write the 10-dimensional spinor Ansatz as

$$
\epsilon(x, y)=\varepsilon(x) \otimes\left[f_{1}(y) \eta_{-}(y)+f_{2}(y) \chi_{-}(y)\right]+\varepsilon^{*}(x) \otimes\left[f_{3}(y) \eta_{+}(y)+f_{4}(y) \chi_{+}(y)\right]
$$

with $f_{i} \in \mathbb{C}$ generic functions to be determined. It is useful to notice however that one can always simplify the above Ansatz, by removing one of the functions by a field redefinition. This is easily proved if one notices that $\phi_{-} \equiv \frac{f_{1} \eta_{-}+f_{2} \chi_{-}}{\left|f_{1}\right|^{2}+\left|f_{2}\right|^{2}}$ has norm one and defines an SU(2) structure together with the orthogonal combination $\psi_{-} \equiv \frac{f_{2}^{*} \eta_{-}-f_{1}^{*} \chi_{-}}{\left|f_{1}\right|^{2}+\left|f_{2}\right|^{2}}$. We remind that for the spinor to be well-behaved everywhere on the solution, we also have to require that the $f_{i}$ functions are globally defined.

We have therefore argued that the most general spinor Ansatz for an $S U(2)$ structure is the Ansatz D (1.5) given in the introduction:

$$
\epsilon(x, y)=a(y) \varepsilon(x) \otimes \eta_{-}(y)+\varepsilon^{*}(x) \otimes\left(b(y) \eta_{+}(y)+c(y) \chi_{+}(y)\right)
$$

One could think about having even more restricting structures on the tangent bundle, of course. The result however would be that the internal space factorizes, often leading to solutions preserving more supersymmetries, and we will therefore not consider it further here.

\section{$2.3 \quad \mathrm{SU}(3)$ and $\mathrm{SU}(2)$ structures}

We have just seen how the existence of different group structures allows for different spinor Ansätze which may lead to new supersymmetric solutions. Since in what follows we are going to make an extensive use of them, we will give some more details on the introduction of a $G$-structure, for $G=S U(3)$ or $G=S U(2)$. Some parts of what follows are also covered in [5, 11, 13].

In the previous section we have learned how globally defined spinors imply a group structure on the tangent bundle of a 6-manifold. Alternatively, one can characterize a $G^{-}$ structure by invariant forms, i.e. tensors which are singlets in the decomposition under $G$. For an $S U(3)$ structure one needs a 2 -form $J$ and a complex 3 -form $\Omega$, as defined in (2.11), 
(2.12), which satisfy the compatibility constraints

$$
J \wedge \Omega=0, \quad J \wedge J \wedge J=\frac{3}{4} i \Omega \wedge \bar{\Omega}
$$

An $S U(2)$ structure requires the existence of a triplet of 2-forms $J^{i}$ and a complex 1-form $w$. The 1-form must lie on an orthogonal space with respect to the one spanned by the 2-forms

$$
w\lrcorner J^{i}=0
$$

and these latter give the volume of a 4-dimensional subspace

$$
J^{i} \wedge J^{j}=0, \text { for } i \neq j, \quad J^{i} \wedge J^{i}=V o l_{4} .
$$

One can also use the metric to raise one of the indices of the 2-forms and obtain a triplet of almost complex structures on the base satisfying

$$
J^{i} J^{j}=-\delta^{i j}-\epsilon^{i j k} J^{k}
$$

These can also be combined again into a 2-form, a complex 3-form and a complex 1-form.

As for the $S U(3)$ structures, one can build the $S U(2)$ structure tensors from combinations of the invariant spinors. We could provide directly the expression analogous to (2.11) and (2.12), but it is instructive to derive the $S U(2)$ tensors starting from an existing $S U(3)$ structure and imposing the existence of some additional independent spinor. In this way it will be clear the difference between the previous analyses of the supersymmetry conditions and the one presented here.

With respect to $S U(3)$ structures, the $S U(2)$ ones are characterized by the existence of an additional globally defined complex vector $w$. One can therefore construct them from existing $S U(3)$ structures by introducing new globally defined spinors as

$$
\chi_{+}=\frac{1}{2} w_{m} \gamma^{m} \eta_{-}, \quad \chi_{-}=\frac{1}{2} \bar{w}_{m} \gamma^{m} \eta_{+},
$$

where it is clear that $\chi_{ \pm}^{*}=\chi_{\mp}$. Assuming that the $\eta_{ \pm}$spinors and the globally defined vector $w$ are canonically normalized, i.e. $\eta_{ \pm}^{\dagger} \eta_{ \pm}=1$ and $w_{m} \bar{w}^{m}=-2$, one obtains more orthogonality relations

$$
\chi_{ \pm}^{\dagger} \chi_{ \pm}=1, \quad \chi_{ \pm}^{\dagger} \eta_{ \pm}=0
$$

where we also used that $w$ is holomorphic with respect to the almost complex structure defined by (2.11) $J_{m}{ }^{n} w_{n}=i w_{m}$.

Combining the information coming from the definition of the $S U(3)$ structure (2.11), (2.12), the definition of the $S U(2)$ spinors (2.20) and the orthogonality properties (2.21) 
we can then obtain the definitions of all the invariant tensors in terms of the fundamental spinors:

$$
\begin{aligned}
\eta_{+}^{T} \gamma^{m} \chi_{+} & =w^{m}, & \eta_{-}^{T} \gamma_{m n} \eta_{+} & =i J_{m n}, \\
\eta_{+}^{T} \gamma_{m n} \chi_{-} & =K_{m n}, & \eta_{-}^{T} \gamma_{m n} \chi_{+} & =\bar{K}_{m n}, \\
\chi_{-}^{T} \gamma_{m n} \chi_{+} & =2 w_{[m} \bar{w}_{n]}-i J_{m n}, & \eta_{+}^{T} \gamma_{m n p} \eta_{+} & =-\Omega_{m n p},
\end{aligned}
$$

where we have introduced the combination $K \equiv J^{2}+i J^{3}$. Moreover, using the identities on the 6-dimensional $\gamma$ matrices and the spinors $\eta$ and $\chi$ reported in the appendix, it can also be checked that $J=J^{1}-\frac{i}{2} w \wedge \bar{w}$ and $\Omega=K \wedge w$.

In doing so, we have made a choice for the embedding of the $S U(2)$ structure inside the $S U(3)$ one. This was done in a somewhat natural way because we have constructed the $S U(2)$ structure starting from an existing $S U(3)$ one. However, it should be clear that given 2 globally defined spinors there is no preferred choice for the one describing the $S U(3)$ structure. Let us then establish how the $S U(2)$ structures are embedded into the $S U(3)$ ones generically. Considering always normalized spinors, there is a $U(1)$ degeneracy of possible spinors defining the $S U(3)$ structure starting from the 2 globally defined spinors describing the $S U(2)$ one:

$$
\beta_{-}=\cos \phi \eta_{-}+\sin \phi \chi_{-} .
$$

Using this normalized spinor one can then define the $S U(3)$ structure as given in (2.11) and (2.12), now with $\beta_{-}$replacing $\eta_{-}$:

$$
\begin{aligned}
J & =\left(1-2 \sin ^{2} \phi\right) J^{1}-\frac{i}{2} w \wedge \bar{w}+\frac{i}{2} \sin 2 \phi(K-\bar{K}), \\
\Omega & =\cos ^{2} \phi K \wedge w+\sin ^{2} \phi \bar{K} \wedge w+i \sin 2 \phi J^{1} \wedge w .
\end{aligned}
$$

It is now clear that the properties of the $S U(3)$ structure depend on the choice of the embedding angle. Among these, the integrability of the complex structure. Although this will not change the fact that the solution be supersymmetric or not, it may change the physical interpretation as there are instances in which there is a natural choice of complex structure which specifies $\phi$. In the following we will consider for definiteness $\phi=0$, i.e. $\beta_{-}=\eta_{-}$as done previously.

\section{Analysis of supersymmetry conditions}

In this section we are going to interpret the supersymmetry equations as conditions on the intrinsic torsion of the internal manifold. This means that we are going to specify some differential constraints on the $S U(2)$ structure tensors defined in (2.22). At the same time we will show that there are some constraints on the 3-form fluxes and dilaton/axion, though 
they will not be as restrictive as those obtained previously using the Ansätze (1.1)-(1.3). For this reason, we list here the general expansion of the fluxes in terms of the $S U(2)$ structures, so that statements on the single modules can be made more precise. The dilaton/axion can be decomposed in 3 pieces

$$
P=p_{1} w+p_{2} \bar{w}+\Pi,
$$

where $w\lrcorner \Pi=0$, for a real $\Pi$. The 3 -form flux is then

$$
\begin{aligned}
G & =g_{30} K \wedge w+g_{21} K \wedge \bar{w}+\tilde{g}_{21} J^{1} \wedge w+J^{1} \wedge V_{1}+w \wedge \bar{w} \wedge V_{2}+ \\
& +w \wedge T_{1}+\bar{w} \wedge T_{2}+g_{12} \bar{K} \wedge w+\tilde{g}_{12} J^{1} \wedge \bar{w}+g_{03} \bar{K} \wedge \bar{w}
\end{aligned}
$$

where the flux components further satisfy

$$
\left.\left.J^{i} \wedge T_{1,2}=0, \quad w\right\lrcorner T_{1,2}=0 \quad \text { and } \quad w\right\lrcorner V_{1,2}=0 .
$$

We can also combine $V_{1}$ and $V_{2}$ so that the primitive and non-primitive parts with respect to $J$ are explicit: $J^{1} \wedge V_{1}+w \wedge \bar{w} \wedge V_{2}=\frac{1}{2} J \wedge\left(V_{1}+2 i V_{2}\right)+\frac{1}{2}\left(J^{1}+\frac{i}{2} w \wedge \bar{w}\right) \wedge\left(V_{1}-2 i V_{2}\right)$. We also notice that $g_{12}, g_{21}$ refer to primitive fluxes with respect to $J$ and $\tilde{g}_{21}, \tilde{g}_{12}$ to nonprimitive ones. Restriction to ISD fluxes can be made by setting

$$
g_{30}=g_{12}=\tilde{g}_{21}=0, \quad T_{2}=0, \quad(1+i J)\left(V_{1}-2 i V_{2}\right)=0=(1-i J)\left(V_{1}+2 i V_{2}\right) .
$$

The supersymmetry equations (2.1), (2.2) are not written in this language. In order to extract the information we want in terms of $S U(2)$ structure conditions we should perform a projection on the full basis of independent spinors and use the relations between the spinor bilinears and the $S U(2)$ structure tensors (2.22). For the case at hand, one should project on the full basis given by $\eta_{ \pm}^{\dagger}, \eta_{ \pm}^{\dagger} \gamma^{m \ldots}$, up to $3 \gamma$ matrices, and the same for the spinors $\chi_{ \pm}$. Luckily, it is not necessary to consider all these projections, because only a subset of them gives independent conditions. As a first fact one can notice that only the projections along $\eta_{ \pm}$are necessary due to the relation (2.20) between $\eta_{ \pm}$and $\chi_{ \pm}$. Then, using group theory, from the $S U(4) \rightarrow S U(3) \rightarrow S U(2)$ decomposition one learns that the only independent objects one can build are $\eta_{ \pm}$and $\gamma^{m} \eta_{ \pm}$. Therefore we will just consider these projections.

We will start analyzing first the dilatino equation (2.2) and then the gravitino equation (2.1) for the free index in the external space and then in the internal space. The first two sets of equations will give us conditions on the fluxes as well as determine the warp-factor and 5-form flux in terms of the 3-form flux. Then, from the internal gravitino we will get the proper differential constraints on the $S U(2)$ structure.

Let us start with the analysis of the dilatino equation. Making explicit use of the D spinor Ansatz, (2.2) reads

$$
\begin{aligned}
\delta \lambda=0 & \Rightarrow \gamma^{m} P_{m}\left[-\varepsilon^{*} \otimes\left(a^{*} \eta_{+}\right)+\varepsilon \otimes\left(b^{*} \eta_{-}+c^{*} \chi_{-}\right)\right]= \\
& =\frac{\kappa}{24 \sqrt{Z}} \gamma^{m n p}\left[\varepsilon \otimes\left(a \eta_{-}\right)-\varepsilon^{*} \otimes\left(b \eta_{+}+c \chi_{+}\right)\right] G_{m n p} .
\end{aligned}
$$


Simple contractions of this equation with $\eta_{ \pm}^{T}$ and $\eta_{ \pm}^{T} \gamma_{m}$ give us two constraints on the fluxes

$$
\begin{aligned}
2 b g_{03}+i c \tilde{g}_{12} & =0, \\
2 b^{*} g_{30}-i c^{*} \tilde{g}_{21} & =0,
\end{aligned}
$$

and equations which determine the dilaton/axion in terms of the components of the 3-form flux as specified in (3.2)

$$
\begin{aligned}
\sqrt{Z} c^{*} p_{1} & =-\kappa a g_{30}, \\
2 \sqrt{Z} a^{*} p_{2} & =\kappa\left(2 c g_{21}+i b \tilde{g}_{12}\right), \\
a^{*}(1+i J) \Pi & =i \frac{\kappa}{4 \sqrt{Z}}\left[b(1+i J)\left(V_{1}+2 i V_{2}\right)+\right. \\
& \left.-c \bar{K}\left(V_{1}-2 i V_{2}\right)\right], \\
b^{*}(1-i J) \Pi+c^{*} K \Pi & =-\frac{\kappa}{4 \sqrt{Z}} i a(1-i J)\left(V_{1}+2 i V_{2}\right) .
\end{aligned}
$$

From now on, when $J, J^{1}$ or $K$ are not separated by wedge products from other forms we understand the associated structures, i.e. $J V \equiv e^{a} J_{a}^{b} V_{b}$. It should be noted from (3.8), (3.9), using (3.6), that the $(3,0)$ and $(0,3)$ components of the 3 -form flux drive a holomorphic and anti-holomorphic part of the dilaton, respectively. Moreover, as noted also in [24], the appearance of a $(0,3)$ flux must be accompanied by a non-primitive $(1,2)$ component.

As a second step one has to analyze the gravitino equation (2.1), when the free index is on the external space $M=\mu$. Using that the 4-dimensional spinor on Minkowski space can be chosen to be constant $\partial_{\mu} \varepsilon(x)=0$, the gravitino equation reads

$$
\begin{aligned}
\delta \psi_{\mu}= & -\frac{1}{8 \kappa \sqrt{Z}} \gamma^{m} \partial_{m} \log Z\left[\gamma_{\mu} \varepsilon \otimes\left(a \eta_{-}\right)-\gamma_{\mu} \varepsilon^{*} \otimes\left(b \eta_{+}+c \chi_{+}\right)\right]+ \\
& +\frac{1}{2} \sqrt{Z} \gamma^{m} \partial_{m} h\left[\gamma_{\mu} \varepsilon \otimes\left(a \eta_{-}\right)+\gamma_{\mu} \varepsilon^{*} \otimes\left(b \eta_{+}+c \chi_{+}\right)\right]+ \\
& +\frac{1}{16 \cdot 6 Z} \gamma^{m n p} G_{m n p}\left[-\gamma_{\mu} \varepsilon^{*} \otimes\left(a^{*} \eta_{+}\right)+\gamma_{\mu} \varepsilon \otimes\left(b^{*} \eta_{-}+c^{*} \chi_{-}\right)\right]=0
\end{aligned}
$$

where we also made explicit the connection of the warped metric, expressing everything in terms of the quantities on the unwarped spaces.

Again, projecting along $\eta_{ \pm}^{T}$ and $\eta_{ \pm}^{T} \gamma_{m}$, we obtain further constraints on the fluxes and determine the warp factor and 5-form flux in terms of the 3-form flux. We also decompose the derivative of the warp factor and the 5-form flux to make explicit the irreducible $S U(2)$ modules

$$
\begin{aligned}
\partial_{m} \log Z & =\sigma w_{m}+\bar{\sigma} \bar{w}_{m}+\Sigma_{m} \\
\partial_{m} h & =\theta w_{m}+\bar{\theta} \bar{w}_{m}+H_{m}
\end{aligned}
$$


where $w\lrcorner \Sigma=w\lrcorner H=0$ and $\Sigma$ and $H$ are real. Plugging these expressions into (3.12) and performing the appropriate projections, not only we get the same flux constraint as (3.7), but also some equations determining the warp factor and the 5 -form flux

$$
\begin{aligned}
\sigma-4 \kappa Z \theta & =\frac{\kappa}{\sqrt{Z}}\left(2 \frac{c^{*}}{a} g_{12}-i \frac{b^{*}}{a} \tilde{g}_{21}\right), \\
\bar{\sigma}+4 \kappa Z \bar{\theta}= & -\frac{2 \kappa a^{*}}{\sqrt{Z} c} g_{03}, \\
(1-i J)[\Sigma-4 \kappa Z H] & =-i \frac{b^{*} \kappa}{2 a \sqrt{Z}}(1-i J)\left(V_{1}+2 i V_{2}\right)+ \\
& +i \frac{c^{*} \kappa}{2 a \sqrt{Z}} K\left(V_{1}-2 i V_{2}\right), \\
(1+i J)[\Sigma+4 \kappa Z H] & +\frac{c}{b} \bar{K}(\Sigma+4 \kappa Z H)= \\
& =i \frac{a^{*} \kappa}{2 b \sqrt{Z}}(1+i J)\left(V_{1}+2 i V_{2}\right) .
\end{aligned}
$$

It should be noted that restriction to ISD fluxes imposes a precise relation between the warp-factor and 5-form flux. Indeed, using (3.4), the right hand side of both (3.15) and (3.17) vanishes and therefore we get that $d \log Z=4 \kappa Z d h$. This relation, using different conventions, was pointed out in [23, 25].

The last, and more demanding, step is given by computing the differential constraints on the $S U(2)$ structure from the analysis of the variation of the internal gravitino $\delta \psi_{m}$. Since the various structures (2.22) are defined in terms of $\eta_{ \pm}$and $\chi_{ \pm}$, in order to obtain differential constraints on them we have to extract $\nabla_{m} \eta_{ \pm}$and $\nabla_{m} \chi_{ \pm}$from $\delta \psi_{m}=0$. This can be done by separating the contributions proportional to $\varepsilon(x)$ from those proportional to $\varepsilon^{*}(x)$ and, in the case of $\nabla \chi_{-}$, by taking appropriate linear combinations of them. The resulting expressions are

$$
\begin{aligned}
\nabla_{m} \eta_{-} & =\left(-\partial_{m} \log a+\frac{i}{2} Q_{m}\right) \eta_{-}-\frac{1}{8} \gamma_{m}^{n} \partial_{n} \log Z \eta_{-}-\frac{\kappa}{2} \gamma^{n} \gamma_{m} Z \partial_{n} h \eta_{-}+ \\
& +\frac{\kappa}{\sqrt{Z}} \frac{b^{*}}{a} G_{p q r}\left(\frac{1}{16 \cdot 6} \gamma_{m}^{p q r}+\frac{3}{32} \gamma^{p q} \delta_{m}^{r}\right) \eta_{-}+ \\
& +\frac{\kappa}{\sqrt{Z}} \frac{c^{*}}{a} G_{p q r}\left(\frac{1}{16 \cdot 6} \gamma_{m}^{p q r}+\frac{3}{32} \gamma^{p q} \delta_{m}^{r}\right) \chi_{-},
\end{aligned}
$$

and

$$
\nabla_{m} \chi_{-}=-\left(\partial_{m} \log c^{*}+\frac{i}{2} Q_{m}\right) \chi_{-}+\left(\frac{b^{*}}{c^{*}} \partial_{m} \log a-\frac{1}{c^{*}} \partial_{m} b^{*}-i \frac{b^{*}}{c^{*}} Q_{m}\right) \eta_{-}+
$$




$$
\begin{aligned}
& -\frac{1}{8} \gamma_{m}{ }^{n} \partial_{n} \log Z \chi_{-}+\frac{\kappa}{2} \gamma^{n} \gamma_{m} Z \partial_{n} h \chi_{-}+\kappa \frac{b^{*}}{c^{*}} \gamma^{n} \gamma_{m} Z \partial_{n} h \eta_{-} \\
& -\frac{\kappa}{\sqrt{Z}} \frac{b^{*}}{a} G_{p q r}\left(\frac{1}{16 \cdot 6} \gamma^{p q r}+\frac{3}{32} \gamma^{p q} \delta_{m}^{r}\right) \chi_{-}+ \\
& -\frac{\kappa}{\sqrt{Z}} \frac{\left(b^{*}\right)^{2} G_{p q r}-a^{2} \bar{G}_{p q r}}{c^{*} a}\left(\frac{1}{16 \cdot 6} \gamma_{m}^{p q r}+\frac{3}{32} \gamma^{p q} \delta_{m}^{r}\right) \eta_{-} .
\end{aligned}
$$

We can now compute $d w$ and $d J^{i}$, but from the orthogonality properties of the $\eta$ and $\chi$ spinors we can also obtain some differential equations specifying the behaviour of the norms $a, b$ and $c$ appearing in (1.5) in terms of the fluxes. First of all, by recalling that $d\left(\eta_{+} \eta_{-}\right)=0=d\left(\chi_{+} \chi_{-}\right)$we get differential conditions on the absolute value of the $a$ and $c$ functions:

$$
\begin{aligned}
2 d \log |a| & =-\kappa Z d h-\left\{\frac{\kappa}{4 \sqrt{Z}} w\left(i \frac{b^{*}}{a} \tilde{g}_{21}-2 i \frac{b}{a^{*}} \tilde{g}_{12}^{*}-2 \frac{c^{*}}{a} g_{12}-4 \frac{c}{a^{*}} g_{03}^{*}\right)+\right. \\
& -\frac{\kappa}{16 \sqrt{Z}}\left[\frac{b^{*}}{a}(J-3 i)\left(V_{1}+2 i V_{2}\right)-\frac{c^{*}}{a} K\left(2 V_{2}-3 i V_{1}\right)\right]+ \\
+ & c . c .\} \\
2 d \log |c| & =\kappa Z d h+\kappa Z\left(\frac{b^{*}}{c^{*}} \bar{K}+\frac{b}{c} K\right) d h+ \\
& -\left\{\frac { \kappa } { 1 6 \sqrt { Z } } \left[\frac{b^{*}}{a}(J+3 i)\left(V_{1}-2 i V_{2}\right)+\frac{a^{*}}{c} K\left(2 V_{2}+3 i V_{1}\right)+\right.\right. \\
& \left.+\frac{\left(b^{*}\right)^{2}}{a c^{*}} \bar{K}\left(2 V_{2}+3 i V_{1}\right)\right]+ \\
& \left.+\frac{\kappa}{4 \sqrt{Z}} w\left(2 \frac{a}{c^{*}} g_{03}^{*}+4 \frac{a^{*}}{c} g_{12}-2 i \frac{b}{a^{*}} \tilde{g}_{12}^{*}-4 \frac{b^{2}}{c a^{*}} g_{21}^{*}\right)+\text { c.c. }\right\} .
\end{aligned}
$$

Then, from $d\left[\chi_{+} \eta_{-}\right]=0$ we get an extra equation for the other function

$$
\begin{aligned}
\frac{1}{c} d b & -\frac{b}{c} d \log a^{*}=i \frac{b}{c} Q+\frac{b}{c} \kappa Z(1-i J) d h-\kappa Z \bar{K} d h+ \\
& +\frac{\kappa}{16 \sqrt{Z}}\left[\frac{a^{*}}{c}(J+3 i)\left(V_{1}+2 i V_{2}\right)+\frac{c^{*}}{a}(J+3 i)\left(V_{1}-2 i V_{2}\right)+\right. \\
& -\frac{b^{2}}{a^{*} c}(J+3 i)\left(\bar{V}_{1}-2 i \bar{V}_{2}\right)+ \\
& \left.+\frac{b^{*}}{a} \bar{K}\left(2 V_{2}+3 i V_{1}\right)+\frac{b}{a^{*}} K\left(2 \bar{V}_{2}+3 i \bar{V}_{1}\right)\right]+ \\
& -\frac{\kappa}{4 \sqrt{Z}} w\left(4 \frac{b}{a^{*}} g_{03}^{*}-2 i \frac{a^{*}}{c} \tilde{g}_{21}+2 i \frac{b^{2}}{a^{*} c} \tilde{g}_{12}^{*}\right)+ \\
& -\frac{\kappa}{4 \sqrt{Z}} \bar{w}\left(2 \frac{b}{a^{*}} g_{12}^{*}+4 \frac{b^{*}}{a} g_{21}-i\left(2 \frac{c^{*}}{a}+\frac{a^{*}}{c}\right) \tilde{g}_{12}+i \frac{b^{2}}{a^{*} c} \tilde{g}_{21}^{*}\right)
\end{aligned}
$$


Let us then turn to the computation of the $S U(2)$ torsion classes. Of course, one could express the various components of the intrinsic torsion completely in terms of the 3-form flux. However, we prefer to use where possible also the warp-factor, the 5 -form flux and the spinor norms $a, b$ and $c$, so as to make the final expressions a bit more concise. In some of the following formulae we will also make use of the almost complex structure $J=J^{1}-\frac{i}{2} w \wedge \bar{w}$ and of the $(3,0)$-form $\Omega=K \wedge w$, to make more intuitive the meaning of some of the various contributions.

The exterior derivative on the globally defined 1 -form $w$ is given by

$$
\begin{aligned}
d w & =\left[-d \log a^{*}-d \log c-\frac{1}{4} d \log Z+2 i \kappa Z J d h+2 \kappa \frac{b}{c} Z K d h+\frac{1}{2} \bar{\sigma} \bar{w}+\right. \\
& -\frac{i \kappa}{8 \sqrt{Z}}\left(-\frac{c^{*}}{a} K\left(\bar{V}_{1}-2 i \bar{V}_{2}\right)+\frac{a^{*}}{c} K\left(V_{1}+2 i V_{2}\right)+\right. \\
& \left.\left.-2 \frac{b}{a^{*}}\left(V_{1}-2 i V_{2}\right)+\frac{c}{a^{*}} \bar{K}\left(V_{1}+2 i V_{2}\right)+4 i \frac{b}{a^{*}}(1+i J) \bar{V}_{2}\right)\right] \wedge w .
\end{aligned}
$$

Performing a straightforward calculation, one obtains in (3.24) other terms proportional to the 2 -forms $J^{1}, K$ and $\bar{K}$. However, by using (3.6), (3.7) and (3.16), (3.17) one can show that all such terms vanish. For instance, the $\bar{K}$ term comes multiplied by $i c \tilde{g}_{21}^{*}+2 b g_{30}^{*}$, which is zero using (3.7). The term proportional to the 2 -form $K$ comes multiplied by $2 \kappa \frac{b}{c} Z \theta^{*}+$ $\frac{1}{4 \sqrt{Z}}\left(i \frac{b^{2}}{a^{*} c} \tilde{g}_{21}^{*}-i \frac{a^{*}}{c} \tilde{g}_{12}+2 \frac{b}{a^{*}} g_{12}^{*}\right)$ and this is again vanishing because of (3.17), (3.18). There is also a contribution proportional to $J^{1}$. This is $2 Z i \kappa \theta^{*}+i \frac{\kappa}{2 \sqrt{Z}}\left(\frac{b^{2}}{c a^{*}} g_{30}^{*}+\frac{a^{*}}{c} g_{03}-\frac{c}{a^{*}} g_{12}^{*}\right)$, which vanishes again using the same equations. Similar simplifications can also be applied to the $w \wedge \bar{w}$ terms to arrive at the final form presented above.

The result in (3.24) imposes already some strong restrictions on the general form of the internal space. The group structures determine the metric of the internal space. For an $S U(2)$ structure defined by $J^{i}$ and $w$ the metric can always be written as

$$
d s_{6}^{2}=d s_{4}^{2}(y)+w \otimes \bar{w}
$$

where both $d s_{4}^{2}$ and $w$ will generically have legs on all possible directions of the cotangent space. However, since from the above result we see that $d w$ is proportional to $w$ itself, we can always define the coordinate differentials so that

$$
w=e^{A(y)} d y_{5}+i e^{B(y)} d y_{6},
$$

for $A$ and $B$ complex functions depending on all the coordinates. The 4 -dimensional part $d s_{4}^{2}$ will still generically depend also on $d y^{5}$ and $d y^{6}$. If, however, the almost product structure $\Pi_{a}^{b}$ defined by $w$ and its dual vector as $\Pi_{a}^{b}=\left(w_{a} \bar{w}^{b}+\bar{w}_{a} w^{b}-\delta_{a}^{b}\right)$ is integrable then the 
6-dimensional metric is further reduced to a block diagonal form

$$
d s_{6}^{2}=\Sigma_{m, n=1}^{4} g_{m n}(y) d y^{m} \otimes d y^{n}+w \otimes \bar{w} .
$$

Unfortunately, (3.24) is not enough to show that $\Pi$ is integrable, unless further constraints are imposed.

We can now complete the analysis by computing the intrinsic torsion contributions coming from the exterior differential on the triplet of 2 -forms. Although we should really compute $d J^{i}$, we will present in the following $d J$ and $d K$. It is then obvious how to extract $d J^{1}$. We just show $d J$ because it is easier to compute and also because it gives direct information on the integrability of the associated almost complex structure $J$. The expression for its exterior differential is

$$
\begin{aligned}
d J & =-J \wedge\left(2 d \log |a|+\frac{1}{2} d \log Z-\kappa Z d h\right)+ \\
& +\frac{i}{16 \sqrt{Z}} \kappa\left\{\frac { b ^ { * } } { a } \left[J \wedge\left(-8 \tilde{g}_{12} \bar{w}-12 \tilde{g}_{21} w-9 V_{1}+5 i J V_{1}-2 i V_{2}+6 J V_{2}\right)+\right.\right. \\
& \left.-4 i w \wedge \bar{w} \wedge(1-i J)\left(V_{1}-2 i V_{2}\right)-16 T^{1} \wedge w-16 g_{21} K \wedge \bar{w}\right]+ \\
& +i \frac{c^{*}}{a}\left[-\frac{1}{2} w \wedge \bar{w} \wedge K\left(V_{1}-10 i V_{2}\right)+8 \tilde{g}_{12} K \wedge \bar{w}+\right. \\
& \left.\left.-J^{1} \wedge K\left(2 V_{2}+5 i V_{1}\right)-J \wedge\left(16 g_{03} \bar{w}+24 g_{12} w\right)\right]- \text { c.c. }\right\}
\end{aligned}
$$

It is worth noting that although there are in principle non vanishing $(3,0)$ or $(0,3)$ components, their coefficient vanish identically using (3.6), (3.7). Finally, we can also compute

$$
\begin{aligned}
d K & =K \wedge\left[-d \log \left(c^{*} a^{*}\right)-i Q-\frac{1}{2} d \log Z\right]+ \\
& +\frac{3 \kappa}{\sqrt{Z}} \Omega\left[-Z^{3 / 2} \theta+\frac{c}{3 a^{*}} g_{03}^{*}+\frac{i}{6} \frac{b}{a^{*}} \tilde{g}_{12}^{*}-\frac{a}{2 c^{*}} g_{03}^{*}\right]+ \\
& +\frac{3 \kappa}{\sqrt{Z}} K \wedge \bar{w}\left[Z^{3 / 2} \bar{\theta}+\frac{c}{2 a^{*}} g_{12}^{*}+\frac{i}{4} \frac{b}{a^{*}} \tilde{g}_{21}^{*}-\frac{i}{6} \frac{b^{*}}{a} \tilde{g}_{12}-\frac{a}{3 c^{*}} g_{12}^{*}+\frac{\left(b^{*}\right)^{2}}{3 c^{*} a} g_{21}\right]+ \\
& +\frac{\kappa}{\sqrt{Z}} J \wedge w\left[\frac{c}{a^{*}} \tilde{g}_{12}^{*}-2 i \frac{b}{a^{*}} g_{21}^{*}+i \frac{b^{*}}{a} g_{12}-\frac{a}{2 c^{*}} \tilde{g}_{12}^{*}+\frac{1}{2} \frac{\left(b^{*}\right)^{2}}{c^{*} a} \tilde{g}_{21}\right]+ \\
& +2 i \kappa \frac{b^{*}}{c^{*}} Z J \wedge(1-i J) d h-i \kappa \frac{b^{*}}{c^{*}} Z \wedge K d h+ \\
& +\frac{\kappa}{16 \sqrt{Z}} J \wedge\left[-6 \frac{a}{c^{*}}(1-i J) \bar{V}_{1}+6 \frac{\left(b^{*}\right)^{2}}{a c^{*}}(1-i J) V_{1}-4 i \frac{a}{c^{*}}(1-i J) \bar{V}_{2}+8 \frac{c}{a^{*}}(1-i J) \bar{V}_{1}+\right. \\
& \left.-4 i \frac{\left(b^{*}\right)^{2}}{a c^{*}}(1-i J) V_{2}+8 \frac{b}{a^{*}} K \bar{V}_{1}-2 \frac{b^{*}}{a} K\left(V_{1}-2 i V_{2}\right)\right]+
\end{aligned}
$$




$$
\begin{aligned}
& +\frac{\kappa}{2 \sqrt{Z}} w \wedge \bar{w} \wedge\left[3 Z^{3 / 2} K d h-\frac{i}{4} \frac{b^{*}}{a} K\left(V_{1}+2 i V_{2}\right)+\frac{3}{8} i \frac{b}{a^{*}} K\left(\bar{V}_{1}+2 i \bar{V}_{2}\right)+\right. \\
& \left.+(1-i J)\left(\frac{3}{8} i \frac{c}{a^{*}}\left(\bar{V}_{1}+2 i \bar{V}_{2}\right)-\frac{i}{2} \frac{a}{c^{*}}\left(\bar{V}_{1}+2 i \bar{V}_{2}\right)+\frac{\left(b^{*}\right)^{2}}{a c^{*}} \frac{i}{2}\left(V_{1}-2 i V_{2}\right)\right)\right]+ \\
& -\frac{\kappa}{\sqrt{Z}} w \wedge\left(\frac{a}{c^{*}} \bar{T}^{2}-\frac{\left(b^{*}\right)^{2}}{a c^{*}} T^{1}\right)+ \\
& +\frac{\kappa}{\sqrt{Z}} \frac{c}{a^{*}} \bar{w} \wedge \bar{T}^{1}+\frac{\kappa}{4 \sqrt{Z}} \frac{c}{a^{*}} J^{1} \wedge(1+i J)\left(\bar{V}_{1}+2 i \bar{V}_{2}\right) .
\end{aligned}
$$

Also this expression has been simplified using the constraints on the fluxes coming from the dilatino and external gravitino. Terms of the form $J \wedge \bar{w}$, which are present in the straightforward calculation, vanish using (3.23) and (3.6), (3.7).

Having the complete form of the intrinsic torsion for the $S U(2)$ structure, we can make some comments on the integrability of the complex structure $J$. It is known that given the $S U(3)$ structure defined by $J$ and $\Omega=K \wedge w$, the torsion classes which describe the integrability of $J$ are $\mathcal{W}_{1}$ and $\mathcal{W}_{2}$, as can be read from

$$
\begin{aligned}
d J & =\frac{3}{4} i\left(\mathcal{W}_{1} \bar{\Omega}-\overline{\mathcal{W}}_{1} \Omega\right)+\mathcal{W}_{3}+J \wedge \mathcal{W}_{4}, \\
d \Omega & =\mathcal{W}_{1} J \wedge J+J \wedge \mathcal{W}_{2}+\Omega \wedge \mathcal{W}_{5} .
\end{aligned}
$$

It is already clear from $d J$ that $\mathcal{W}_{1}=0$, since there is no $(0,3)$ or $(3,0)$ form in (3.28). Moreover, $d w$ is always proportional to $w$ and therefore the only obstruction to the integrability of $J$ can only be given by the $(1,2)$ contributions coming from $d K$. By inspection of (3.29) it is easy to see that only its last line contains objects of $(1,2)$ type. From there we conclude that

$$
\mathcal{W}_{2}=\frac{\kappa}{4 \sqrt{Z}} \frac{c^{*}}{a}\left(8 i \bar{T}^{1}-w \wedge(1+i J)\left(\bar{V}_{1}+2 i \bar{V}_{2}\right)\right)
$$

and therefore $J$ is an integrable complex structure only if the following additional constraints on the 3-form flux are imposed:

$$
T^{1}=0, \quad(1-i J)\left(V_{1}-2 i V_{2}\right)=0
$$

It should be noted that these constraints are somehow orthogonal to the ISD requirement (3.4) and imply that if such condition is imposed the only primitive flux allowed is $g_{21}$. Of course, this is just one of the possible almost complex structures described by the $S U(2)$ structure. It is anyway possible to show that for any choice of phase in (2.24) $\mathcal{W}_{1}$ remains zero. For $\mathcal{W}_{2}$ we get an involved expression depending on $T_{1}, T_{2}, V_{1}$ and $V_{2}$, which does not seem to vanish for any choice of phase. As noted also by Frey, in the $c=0$ limit (3.32) vanishes, i.e. $\mathcal{W}_{1}=\mathcal{W}_{2}=0$, and therefore the complex structure is always integrable. 


\section{Solutions}

In principle, from the expressions we obtained in the previous section for the $S U(2)$ structure, one should be able to provide classes of manifolds having the right properties to be supersymmetric backgrounds to type IIB supergravity. Unfortunately, as it is clear by inspection of (3.24), (3.28) and (3.29), although $d w$ and $d J^{i}$ give us some interesting information on the general properties of the internal manifold, they are not very illuminating on how to reproduce them in explicit examples. We leave for the future a more detailed analysis of these conditions as well as the presentation of more examples. Here we will anyway provide some very simple classes of manifolds which have the right group structures. First, we will make contact with the known results obtained for the type A (1.1) and type B Ansatz (1.2). Then we will provide a new class of supersymmetric configurations which admit also $(0,3)$ flux.

\subsection{Known limits: type B and type A solutions}

In order to make contact with the general solution using the type B Ansatz provided in [26] we should set $b=c=0$. Of course, since in this case there is just one spinor on the internal manifold, we cannot discuss anymore constraints on the $S U(2)$ structure but only on the $S U(3)$ one. This means that it is not correct to use the form of $P$ and $G$ as in (3.1) and (3.2). We can however assume that form locally, so that we can check our previous conditions. Moreover one should be careful in setting $b=c=0$ in the differential conditions derived from the internal gravitino, since it would not be consistent, but one should rather reconsider (2.1) in the appropriate conventions.

From the dilatino and external gravitino conditions one gets that

$$
\begin{aligned}
(1+i J) \Pi=p_{2}=0, & & g_{03}=g_{30}=\tilde{g}_{21}=\tilde{g}_{21}=\left(V_{1}+2 i V_{2}\right), \\
\sigma=4 \kappa Z \theta, & & (1-i J)(\Sigma-4 \kappa Z H)=0 .
\end{aligned}
$$

The first condition means that an holomorphic dilaton is allowed, whereas its anti-holomorphic part should vanish $P^{(0,1)}=0$. It is also evident what mentioned in the introduction that such Ansatz does not allow for any $(3,0)$ or $(0,3)$ fluxes. Moreover, the 3 -form flux is also constrained so that the non-primitive $(1,2)_{N P}$ and $(2,1)_{N P}$ parts are vanishing as well. Due to reality of both the warp factor $Z$ and the 5 -form flux $h$, the second equation tells us that

$$
d \log Z=4 \kappa Z d h, \quad \Rightarrow \quad h=-\frac{1}{4 \kappa Z},
$$

which is a mentioned feature of all ISD backgrounds. From the internal gravitino then one gets that

$$
2 d \log |a|=-\kappa Z d h, \quad \Rightarrow \quad|a|=Z^{-1 / 8}
$$


If we now decompose the function in front of the spinor into norm and phase, $a=|a| e^{i \phi_{a}}$, and use (4.4), the differential condition on the spinor which is left is

$$
\nabla_{m} \eta_{-}=\frac{i}{2}\left(Q_{m}-2 \partial_{m} \phi_{a}\right) \eta_{-},
$$

which is equivalent to (2.19) of [26]. This now implies the differential constraints on the $S U(3)$ structure, which read

$$
d J=0, \quad d \Omega=-i \Omega \wedge\left(Q-2 d \phi_{a}\right) .
$$

For consistency from (2.1) we also obtain that

$$
G^{(1,2)}=0,
$$

which finally leaves only a $(2,1)$ flux and primitive. Since $Q$ is a $U(1)$ connection, (4.6) implies that the internal space is a Kähler manifold with vanishing first Chern class, i.e. a Calabi-Yau manifold, but equipped with a metric which is not the usual Ricci-flat one. If we want to use the standard Ricci-flat metric, we can reabsorb the extra connection in the phase $\phi_{a}$, by imposing

$$
d \phi_{a}=-\frac{1}{4} J d \log \left(1-|B|^{2}\right) .
$$

This is possible because in (4.6) only the anti-holomorphic part of $Q$ appears and this latter can be integrated to $i / 2 \bar{\partial} \log \left(1-|B|^{2}\right)$. Once (4.8) is imposed, we obtain the usual Calabi-Yau conditions, namely having a Kähler form and a closed holomorphic 3-form

$$
d J=d \Omega=0 .
$$

The final result is the known fact that to preserve supersymmetry with the B Ansatz (1.2) one needs a $(2,1)_{P}$ flux and the internal manifold must be conformally Calabi-Yau.

It should be noted that by choosing $c \neq 0$ and $a=0$ one gets an anti-holomorphic dilaton, instead of holomorphic, and the flux is $(1,2)_{P}$. Moreover the signs in (4.3), (4.4) are reversed.

For what concerns the type A Ansatz, we will briefly show that we can match the conditions coming from the analysis of the common sector of all supergravity theories [3, 5]. For this reason we assume here that the axion is constant, which means that $P=\frac{1}{2} d \phi$ and $Q=0$, that the 5 -form flux is vanishing, $h=0$, and that the only non-vanishing 3 -form is the NS one, which also means that $\bar{G}=G$. In the spinor Ansatz we have to impose that $c=0$ and $b=a^{*}$, which means again that we have to be very careful in taking the limit of our analysis, since only an $S U(3)$ structure is allowed. 
The dilatino equation kills immediately the $(3,0)$ and $(0,3)$ parts of the flux as expected, $g_{30}=g_{03}=0$. Then we obtain that the non-primitive $(2,1)+(1,2)$ components of $G$ are related to the dilaton as $G^{(2,1)_{N P}+(1,2)_{N P}}=2 \frac{\sqrt{Z}}{\kappa} J \wedge J P$. From the external gravitino we obtain the link between warp-factor and the same components of the flux, which then translate into a relation between dilaton and warp-factor $d \log Z=d \phi$. Finally, from the internal gravitino we obtain a relation between the norm of the spinor and again the $(2,1)_{N P}+(1,2)_{N P}$ parts of the flux, $d \log |a|=-\frac{1}{8} d \phi$, and the differential conditions on the group structures

$$
\begin{aligned}
d J & =J \wedge d \phi-\kappa * G \\
d \Omega & =-\frac{1}{2} \Omega \wedge d \phi .
\end{aligned}
$$

In (4.10) the dual of the 3-form flux can be reconstructed by using the duality properties of the various flux components as discussed in the appendix (A.22)-(A.24).

\subsection{A simple supersymmetric configuration with $(0,3)$ flux}

We now work out a simple example of a configuration which preserves $\mathcal{N}=1$ supersymmetry and has a non-vanishing $(0,3)$ component of the 3 -form flux. To simplify at most the framework in which we work we will assume that $b=0$ and that the flux is given only by

$$
G=g_{03} \bar{K} \wedge \bar{w}+g_{12} \bar{K} \wedge w
$$

Of course, the Hodge decomposition is done with respect to the complex structure (2.11). However, with respect to the general definitions (2.25) one sees that the 3 -form flux contains a non-vanishing $(0,3)$ flux for any phase such that $\cos \phi \neq 0$ and in that case it contains a non-vanishing $(3,0)$ part. We will also assume that the 5 -form is vanishing, i.e. $h=0$. From its Bianchi identity (2.5) we get that

$$
d F_{5}=i \frac{\kappa}{8} G \wedge \bar{G}=-i \frac{\kappa}{8}\left(\left|g_{03}\right|^{2}-\left|g_{12}\right|^{2}\right) \Omega \wedge \bar{\Omega}=0
$$

and this implies that

$$
\left|g_{03}\right|=\left|g_{12}\right|
$$

The conditions following from the dilatino equation give

$$
\left.p_{1}=p_{2}=0, \quad(1+i J) \Pi=0=K \Pi, \quad \Leftrightarrow \quad P^{(0,1)}=0, \text { and } \bar{w}\right\lrcorner P=0 .
$$

This means that the dilaton admits a non-trivial $(1,0)$ component on the base space, and possibly it is holomorphic with respect to the complex coordinates defined by the natural complex structure when this latter is integrable. 
From the external components of the gravitino, one gets (using the fact that $d \log Z$ is real)

$$
\sigma=-\frac{2 \kappa a}{\sqrt{Z} c^{*}} g_{03}^{*}=\frac{2 \kappa c^{*}}{\sqrt{Z} a} g_{12},
$$

and $H=0$ as requires compatibility with our assumptions. Therefore

$$
d \log Z=\frac{2 \kappa}{\sqrt{Z}}\left(\frac{c^{*}}{a} g_{12} w+\frac{c}{a^{*}} g_{12}^{*} \bar{w}\right) .
$$

From the internal gravitino we finally get conditions on the normalizations of the spinors defining the $S U(2)$ structure:

$$
\begin{aligned}
& 2 d \log |a|=\left(\frac{1}{4}-\frac{1}{2}\left|\frac{c}{a}\right|^{2}\right) d \log Z, \\
& 2 d \log |c|=\left(\frac{1}{4}-\frac{1}{2}\left|\frac{a}{c}\right|^{2}\right) d \log Z .
\end{aligned}
$$

A possible solution to such conditions is given by choosing $a$ and $c$ real and

$$
a=c=Z^{-1 / 8} .
$$

The differential conditions defining the torsion classes are then

$$
\begin{aligned}
d w & =\frac{1}{2} d \log Z \wedge w, \\
d J^{1} & =0, \\
d K & =i K \wedge Q,
\end{aligned}
$$

where we used (4.19) and (4.20), and the inherited $S U(3)$ structure satisfies $d J=0, d \Omega=$ $-\frac{1}{2} \Omega \wedge(d \log Z+2 i Q)$. For such examples the product structure $\Pi_{a}^{b}=\left(w_{a} \bar{w}^{b}+\bar{w}_{a} w^{b}-\delta_{a}^{b}\right)$ is integrable and $Q$ depends only on the coordinates of the base. The total space is therefore a direct product of a Calabi-Yau 2-fold and $\mathbb{R}^{2}$. Finally, we impose the $G$ Bianchi identity (2.4). A solution, which ensures also the equations of motion at least for constant dilaton, is given by

$$
g_{12}=-g_{03}^{*}=Z^{-1 / 2} f\left(y_{5}+i y_{6}\right),
$$

where $f$ is an arbitrary holomorphic function depending only on the fiber coordinates. From (4.22) one can see that the almost complex structure is actually integrable and that therefore the axion-dilaton $P$ is holomorphic in the appropriate complex coordinates defined by $J$.

For the case of a constant dilaton/axion the solution then reads

$$
\begin{aligned}
d s_{10}^{2} & =Z^{-1 / 2}\left(d x_{0}^{2}-d x_{1}^{2}-d x_{2}^{2}-d x_{3}^{2}\right)-Z^{1 / 2} d s_{K 3}^{2}\left(y_{1 \ldots 4}\right)-Z\left(d y_{5}^{2}+d y_{6}^{2}\right) \\
G & =Z^{-1 / 2}\left(-f^{*}(\bar{y}) \bar{\Omega}+f(y) K \wedge w\right) \\
\epsilon_{10} & =Z^{-1 / 8}\left(\varepsilon \otimes \eta_{-}+\varepsilon^{*} \otimes \chi_{+}\right) .
\end{aligned}
$$


Consistency further imposes that

$$
Z\left(y_{5}, y_{6}\right)=\kappa^{2}\left[\int f(y) d y+\int f^{*}(\bar{y}) d \bar{y}\right]^{2},
$$

where $y \equiv y_{5}+i y_{6}$. It is worth noting that the warp factor is a function which cannot be consistently defined on a torus and this implies that the form presented above is correct only for a non-compact background. This agrees with the fact that without adding sources one cannot obtain compact solutions for nontrivial fluxes [34, 35.

\section{Comments}

The conditions on the fluxes and on the $S U(2)$ structures of the internal manifold given here followed simply by the analysis of the supersymmetry conditions. The equations of motion will further restrict the possible solutions. Under quite general assumptions it has been shown in 25] that for compact backgrounds the allowed 3-form fluxes are of the ISD type. So far only solutions with $(2,1)$ and primitive flux have been given when also supersymmetry is required. From the analysis we have presented, though, it should be possible to compactify on 6 -manifolds also in the presence of $(0,3)$ and $(1,2)$ non-primitive fluxes, still preserving supersymmetry.

When the internal manifold is not compact, no further restriction on the Hodge type of the 3-form fluxes follows from the equations of motion. Holographic descriptions of renormalization group flows are in this class of solutions and indeed, by the analysis of [24, 36, 28, simple perturbations of the $A d S_{5} \times S^{5}$ metric by 3-form fluxes generically turn on all possible types of the same flux. The flow presented in [37] is especially interesting. There a confining $\mathcal{N}=1$ gauge theory is obtained by adding a mass perturbation to $\mathcal{N}=4$ Yang-Mills theory. It was argued that such solution could be described on the dual side by a type IIB configuration which interpolates between a vacuum with only D3 branes and one where the D3 branes are polarized into D5/NS5 branes wrapped on a 2-sphere of the internal space. The supersymmetry conditions for this flow have been studied up to second order in the perturbation parameter [24] (see also [26, 36, 27]. Again, the standard A, B or C supersymmetry Ansätze are not enough to describe it and it can be argued that it must then fall into our description. A detailed analysis of the flow is currently under investigation [38] and it shows that the internal manifold presents an $S U(2)$ structure and the solution of the supersymmetry equations involves again the D type Ansatz (1.5).

The possibility of having general types of fluxes compatible with supersymmetry is very important in the context of the gauge/gravity correspondence. It has been shown [39, 40, 25] that the superpotential of gauge theories dual to IIB strings on Calabi-Yau manifolds in the 
presence of 3-form fluxes is given by $W=\int G \wedge \Omega$. From the field theory point of view then there is no reason for $W$ to vanish even for supersymmetric solutions. Supersymmetric configurations are only specified by $\partial_{I} W=0$, where $I$ runs on the moduli of the theory. This however cannot happen if the standard solutions of type IIB theory are used, since the flux contains only $(2,1)$ fluxes and therefore $W=0$. The framework presented here on the other hand allows for non-vanishing $(0,3)$ fluxes, so that $G \wedge \Omega \neq 0$ and therefore we it should be employed to obtain dual descriptions of those gauge theories which allow for non-vanishing $W$.

Recently, in [28, Pilch and Warner have discussed a class of "dielectric" $\mathcal{N}=1$ solutions of type IIB supergravity which include the dual of the Leigh-Strassler flow. Their approach is similar to the one presented here, though they impose from the beginning a definite Ansatz for the metric and set to zero the dilaton/axion. Having a purely holomorphic 2 -form potential, it is obvious that it may have non-vanishing $(3,0)$ components. It is therefore interesting to understand how their solution can fit into our discussion. Following the explanations given in the introduction, their spinor Ansatz must not be of the A, B, or $\mathrm{C}$ type and indeed, as they notice, it does not fit in those schemes. It can instead be put in the form of the type D Ansatz (1.5). The projector on the 10-dimensional spinor presented in 28 , is

$$
\frac{1}{2}\left(1+i \Gamma^{0} \Gamma^{1} \Gamma^{2} \Gamma^{3} \Gamma^{4}\left(\cos \beta-e^{-i \phi} \sin \beta \Gamma^{7} \Gamma^{10} *\right)\right) \epsilon=\epsilon,
$$

where the star denotes complex conjugation and we used their numbering for the $\Gamma$ matrices. It is a simple exercise to show that (1.5) satisfies this constraint assuming that $a=A(1+$ $\cos \beta), b=0, c=A e^{-i \phi} \sin \beta$ and $\chi_{+}=\gamma^{7} \gamma^{10} \eta_{+}$, for a so far undetermined function $A$. It can also be shown that their choice of complex structure is not the standard one built from $\eta$. We will return elsewhere on the precise embedding of the full solution given in [28] into the torsion conditions provided in this paper as well as on other solutions which fit into our scheme 41].

\section{Acknowledgments}

We would like to thank G.L. Cardoso, G. Curio, D. Lüst and N. Prezas for enlightening discussions. This work is partially supported by the European Community's Human Potential Programme under contract HPRN-CT-2000-00131 Quantum Spacetime.

\section{A Useful formulae}

For the 10-dimensional notations and conventions we follow 30]. This means that the 10dimensional $\Gamma$-matrices satisfy the Clifford algebra $\left\{\Gamma^{M}, \Gamma^{N}\right\}=2 \eta^{M N}$ where $\eta=\operatorname{diag}\{+-$ 
$-\ldots-\}$ and the duality relation is

$$
\Gamma^{A_{1} \ldots A_{p}}=-(-1)^{p(p-1) / 2} \frac{1}{(10-p) !} \epsilon^{A_{1} \ldots A_{10}} \Gamma_{A_{p+1} \ldots A_{10}} \Gamma^{11},
$$

where we denote by $A_{1}, \ldots A_{10}$ flat 10 -dimensional indices. In a concrete representation one can choose $\Gamma^{0}$ antisymmetric and imaginary, with the others symmetric and imaginary. $\Gamma^{11}$ is then symmetric and real. When performing the reduction on the solution, the 10dimensional $\Gamma$ matrices get split as

$$
\Gamma^{\alpha}=\gamma^{\alpha} \otimes 1, \quad \Gamma^{a}=\gamma^{5} \otimes \gamma^{a}, \quad \Gamma^{11}=\gamma^{5} \otimes \gamma^{7},
$$

where we have used the flat indices on Minkowski space-time $\alpha=0, \ldots, 3$ and on the 6 dimensional internal space $d s_{6}^{2} a=1, \ldots, 6$. We are also assuming that $\gamma^{5}=i \gamma^{0123}$ and then the 6-dimensional duality relation is

$$
\gamma_{m_{1} \ldots m_{k}}=-i \frac{(-1)^{\frac{k(k-1)}{2}}}{(6-k) !} \epsilon_{m_{1} \ldots m_{6}} \gamma^{m_{k+1} \ldots m_{6}} \gamma^{7} .
$$

Again, in a concrete representation the 6-dimensional $\gamma$ matrices are antihermitian $\left(\gamma^{m}\right)^{\dagger}=$ $-\gamma^{m}$ and purely real $\left(\gamma^{m}\right)^{*}=\gamma^{m}$.

In the text we have assumed that the globally defined spinors defining the $S U(3)$ and $S U(2)$ structures are chiral and satisfy $\eta_{ \pm}^{*}=\eta_{\mp}$. This happens for instance in a concrete representation where

$$
\gamma^{7}=\left(\begin{array}{cc}
0 & -i \\
i & 0
\end{array}\right), \quad \eta_{+}=\left(\begin{array}{c}
-i \xi \\
\xi
\end{array}\right), \quad \eta_{-}=\left(\begin{array}{c}
i \xi \\
\xi
\end{array}\right),
$$

for $\xi^{*}=\xi$ an arbitrary 4-dimensional spinor. It may be useful to have an explicit representation of the $\gamma$ matrices satisfying all the above properties. One possibility is for instance given by

$$
\begin{gathered}
\gamma_{18}^{1}=\gamma_{23}^{1}=\gamma_{54}^{1}=\gamma_{76}^{1}=1, \\
\gamma_{41}^{2}=\gamma_{72}^{2}=\gamma_{36}^{2}=\gamma_{58}^{2}=1, \\
\gamma_{17}^{3}=\gamma_{42}^{3}=\gamma_{53}^{3}=\gamma_{68}^{3}=1, \\
\gamma_{31}^{4}=\gamma_{28}^{4}=\gamma_{64}^{4}=\gamma_{57}^{4}=1, \\
\gamma_{25}^{5}=\gamma_{43}^{5}=\gamma_{61}^{5}=\gamma_{78}^{5}=1, \\
\gamma_{12}^{6}=\gamma_{38}^{6}=\gamma_{65}^{6}=\gamma_{74}^{6}=1 .
\end{gathered}
$$

Useful rearrangements when computing the conditions deriving from supersymmetry are

$$
\eta_{ \pm} \eta_{ \pm}^{\dagger}=\frac{1}{8}\left(1 \pm \gamma^{7}\right) \pm \frac{i}{16} J_{m n} \gamma^{m n}\left(1 \pm \gamma^{7}\right),
$$


and

$$
\eta_{+} \eta_{+}^{T}=\frac{1}{48} \Omega_{m n p} \gamma^{m n p}, \quad \eta_{-} \eta_{-}^{T}=\frac{1}{48} \bar{\Omega}_{m n p} \gamma^{m n p} .
$$

We list here all the useful relations (transpose is understood where needed to build the appropriate tensor combination) for the 1 -forms:

$$
\begin{array}{ll}
\eta_{+} \gamma^{m} \chi_{+}=w^{m}, & \chi_{+} \gamma^{m} \eta_{+}=-w^{m}, \\
\eta_{-} \gamma^{m} \chi_{-}=\bar{w}^{m}, & \chi_{-} \gamma^{m} \eta_{-}=-\bar{w}^{m},
\end{array}
$$

2-forms:

$$
\begin{array}{cl}
\eta_{-} \gamma_{m n} \eta_{+}=i J_{m n}, & \eta_{+} \gamma_{m n} \eta_{-}=-i J_{m n}, \\
\eta_{+} \gamma_{m n} \chi_{-}=K_{m n}, & \eta_{-} \gamma_{m n} \chi_{+}=\bar{K}_{m n}, \\
\chi_{-} \gamma_{m n} \chi_{+}=2 w_{[m} \bar{w}_{n]}-i J_{m n}, & \chi_{+} \gamma_{m n} \chi_{-}=-2 w_{[m} \bar{w}_{n]}+i J_{m n},
\end{array}
$$

3-forms:

$$
\begin{array}{cl}
\eta_{+} \gamma_{m n p} \eta_{+}=-\Omega_{m n p}, & \eta_{-} \gamma_{m n p} \eta_{-}=-\bar{\Omega}_{m n p}, \\
\chi_{-} \gamma_{m n p} \chi_{-}=-3 K_{[m n} \bar{w}_{p]}, & \chi_{+} \gamma_{m n p} \chi_{+}=-3 \bar{K}_{[m n} w_{p]}, \\
\eta_{-} \gamma_{m n p} \chi_{-}=3 i J_{[m n} \bar{w}_{p]}, & \eta_{+} \gamma_{m n p} \chi_{+}=-3 i J_{[m n} w_{p]},
\end{array}
$$

4-forms:

$$
\eta_{-} \gamma_{m n p q} \eta_{+}=\eta_{+} \gamma_{m n p q} \eta_{-}=-3 J_{[m n} J_{p] q}
$$

It is also interesting to point out the following duality relations

$$
\begin{aligned}
\epsilon_{i j k}{ }^{a b c} \Omega_{a b c}=-6 i \Omega_{i j k}, & \epsilon_{i j k}{ }^{a b c} \bar{\Omega}_{a b c}=6 i \bar{\Omega}_{i j k}, \\
\epsilon_{a b c}{ }^{m n p} \bar{K}_{m n} w_{p}=-6 i \bar{K}_{[a b} w_{c]}, & \epsilon_{a b c}{ }^{m n p} K_{m n} \bar{w}_{p}=6 i K_{[a b} \bar{w}_{c]}, \\
\epsilon_{a b c}{ }^{m n p} J_{m n} w_{p}=-6 i J_{[a b} w_{c]}, & \epsilon_{a b c}{ }^{m n p} J_{m n} \bar{w}_{p}=6 i J_{[a b} \bar{w}_{c]} .
\end{aligned}
$$

These equations show that given a 3 -form, the $(3,0)+(1,2)_{P}+(2,1)_{N P}$ parts are selfdual

whereas the $(0,3)+(2,1)_{P}+(1,2)_{N P}$ are anti-selfdual (Here $P$ stands for primitive with respect to $J$ and $N P$ for non-primitive).

\section{A.1 SU(2) structure and Dirac spinors}

A further alternative possibility to define the $S U(2)$ structure is by means of a single Dirac spinor

$$
\zeta=\frac{1}{\sqrt{2}}\left(\eta_{-}+\chi_{+}\right)=\frac{1}{\sqrt{2}}\left(1+\frac{1}{2} w_{m} \gamma^{m}\right) \eta_{-}
$$


This spinor does not have a definite chirality and $\zeta^{*}$ is independent from $\zeta$. The structure then follows from

$$
\begin{aligned}
1 & =\zeta^{\dagger} \zeta, \\
w_{m} & =\zeta^{\dagger} \gamma_{m}\left(1+\gamma^{7}\right) \zeta, \\
J_{m n}^{1} & =i \zeta^{\dagger} \gamma_{m n} \zeta, \\
w_{[m} \bar{w}_{n]} & =\zeta^{\dagger} \gamma_{m n} \gamma^{7} \zeta, \\
K_{m n} & =-\zeta^{\dagger} \gamma_{m n} \gamma^{7} \zeta^{*} .
\end{aligned}
$$

The other combinations vanish if they cannot be obtained by the above ones by complex conjugation.

\section{References}

[1] J. P. Gauntlett, D. Martelli, S. Pakis and D. Waldram, G-structures and wrapped NS5branes, hep-th/0205050.

[2] S. Gurrieri, J. Louis, A. Micu and D. Waldram, Mirror symmetry in generalized CalabiYau compactifications, Nucl. Phys. B654 (2003) 61-113 hep-th/0211102.

[3] G. L. Cardoso, G. Dall'Agata, D. Lüst, P. Manousselis and G. Zoupanos, NonKaehler string backgrounds and their five torsion classes, Nucl. Phys. B652 (2003) 5-34 hep-th/0211118.

[4] J. P. Gauntlett and S. Pakis, The geometry of D = 11 Killing spinors, JHEP 04 (2003) 039 hep-th/0212008.

[5] J. P. Gauntlett, D. Martelli and D. Waldram, Superstrings with intrinsic torsion, hep-th/0302158.

[6] P. Kaste, R. Minasian and A. Tomasiello, Supersymmetric M-theory compactifications with fluxes on seven-manifolds and G-structures, JHEP 07 (2003) 004 hep-th/0303127.

[7] D. Martelli and J. Sparks, G-structures, fluxes and calibrations in M-theory, Phys. Rev. D68 (2003) 085014 hep-th/0306225.

[8] J. P. Gauntlett, J. B. Gutowski and S. Pakis, The geometry of D $=11$ null Killing spinors, JHEP 12 (2003) 049 hep-th/0311112. 
[9] K. Behrndt and C. Jeschek, Fluxes in M-theory on 7-manifolds: G-structures and superpotential, hep-th/0311119.

[10] S. Fidanza, R. Minasian and A. Tomasiello, Mirror symmetric SU(3)-structure manifolds with NS fluxes, hep-th/0311122.

[11] G. Dall'Agata and N. Prezas, $N=1$ geometries for $M$-theory and type IIA strings with fluxes, Phys. Rev. D69 (2004) 066004 hep-th/0311146.

[12] K. Behrndt and M. Cvetic, General $N=1$ supersymmetric flux vacua of (massive) type IIA string theory, hep-th/0403049.

[13] J. P. Gauntlett, D. Martelli, J. Sparks and D. Waldram, Supersymmetric Ad $S_{5}$ solutions of M-theory, hep-th/0402153.

[14] A. R. Frey, Warped Strings: Self-dual Flux and Contemporary Compactifications. PhD thesis, Department of Physics University of California, Santa Barbara, 2003. hep-th/0308156.

[15] G. Dall'Agata, String compactifications with fluxes, HU-EP-04/03, Class. Quant. Grav. 21 (2004), 1-21.

[16] A. Strominger, Superstrings with torsion, Nucl. Phys. B274 (1986) 253.

[17] G. Papadopoulos and A. A. Tseytlin, Complex geometry of conifolds and 5-brane wrapped on 2- sphere, Class. Quant. Grav. 18 (2001) 1333-1354 hep-th/0012034.

[18] S. Kachru, M. B. Schulz, P. K. Tripathy and S. P. Trivedi, New supersymmetric string compactifications, JHEP 03 (2003) 061 hep-th/0211182.

[19] K. Becker, M. Becker, K. Dasgupta and P. S. Green, Compactifications of heterotic theory on non-Kaehler complex manifolds. I, JHEP 04 (2003), no. 007052 hep-th/0301161.

[20] K. Becker, M. Becker, P. S. Green, K. Dasgupta and E. Sharpe, Compactifications of heterotic strings on non-Kaehler complex manifolds. II, Nucl. Phys. B678 (2004) 19-100 hep-th/0310058.

[21] K. Becker and M. Becker, M-Theory on eight-manifolds, Nucl. Phys. B477 (1996) 155167 hep-th/9605053.

[22] A. Kehagias, New type IIB vacua and their F-theory interpretation, Phys. Lett. B435 (1998) 337-342 hep-th/9805131. 
[23] S. S. Gubser, Supersymmetry and F-theory realization of the deformed conifold with three-form flux, hep-th/0010010.

[24] M. Grana and J. Polchinski, Supersymmetric three-form flux perturbations on $A d S_{5}$, Phys. Rev. D63 (2001) 026001 hep-th/0009211.

[25] S. B. Giddings, S. Kachru and J. Polchinski, Hierarchies from fluxes in string compactifications, Phys. Rev. D66 (2002) 106006 hep-th/0105097.

[26] M. Grana and J. Polchinski, Gauge / gravity duals with holomorphic dilaton, Phys. Rev. D65 (2002) 126005 hep-th/0106014.

[27] A. R. Frey and M. Grana, Type IIB solutions with interpolating supersymmetries, Phys. Rev. D68 (2003) 106002 hep-th/0307142.

[28] K. Pilch and N. P. Warner, $N=1$ supersymmetric solutions of IIB supergravity, hep-th/0403005.

[29] J. H. Schwarz and P. C. West, Symmetries and transformations of chiral $N=2 D=10$ supergravity, Phys. Lett. B126 (1983) 301.

[30] J. H. Schwarz, Covariant field equations of chiral N=2 D = 10 supergravity, Nucl. Phys. B226 (1983) 269.

[31] G. Dall'Agata, K. Lechner and D. P. Sorokin, Covariant actions for the bosonic sector of $D=10$ IIB supergravity, Class. Quant. Grav. 14 (1997) L195-L198 hep-th/9707044.

[32] G. Dall'Agata, K. Lechner and M. Tonin, $D=10, N=I I B$ supergravity: Lorentzinvariant actions and duality, JHEP 07 (1998) 017 hep-th/9806140.

[33] S. Chiossi and S. Salamon, The intrinsic torsion of $S U(3)$ and $G_{2}$ structures, in Differential geometry, Valencia, 2001, pp. 115-133. World Sci. Publishing, River Edge, NJ, 2002. math.DG/0202282.

[34] B. de Wit, D. J. Smit and N. D. Hari Dass, Residual supersymmetry of compactified d = 10 supergravity, Nucl. Phys. B283 (1987) 165.

[35] J. M. Maldacena and C. Nunez, Supergravity description of field theories on curved manifolds and a no go theorem, Int. J. Mod. Phys. A16 (2001) 822-855 hep-th/0007018.

[36] O. Aharony, B. Kol and S. Yankielowicz, On exactly marginal deformations of $N=4$ SYM and type IIB supergravity on $A d S_{5} \times S^{5}$, JHEP 06 (2002) 039 hep-th/0205090. 
[37] J. Polchinski and M. J. Strassler, The string dual of a confining four-dimensional gauge theory, hep-th/0003136.

[38] G. L. Cardoso, G. Curio, G. Dall'Agata and D. Lüst, "in preparation.".

[39] S. Gukov, C. Vafa and E. Witten, CFT's from Calabi-Yau four-folds, Nucl. Phys. B584 (2000) 69-108 hep-th/9906070.

[40] S. Gukov, Solitons, superpotentials and calibrations, Nucl. Phys. B574 (2000) 169-188 hep-th/9911011.

[41] J. F. G. Cascales and A. M. Uranga, M5-brane geometries, T-duality and fluxes, JHEP 01 (2004) 021 hep-th/0307156. 